\title{
The Application of Artificial Intelligence Technology in Smart Energy
}

\author{
XIAOTAO JIANG ${ }^{1 *}$, MING XIN ZHAO ${ }^{2}$, WEI LIU ${ }^{3}$ and RUO BING XU ${ }^{4}$ \\ ${ }^{1,4}$ State Gird Nanjing Power Supply Company, Nanjing, Jiangsu, 210008, China \\ ${ }^{2,3}$ Department, China Electric Power Research Institute, Beijing, 100192, China
}

\begin{abstract}
At present, the development of energy system tends to be clean and intelligent. China has upgraded the development of smart energy to national strategy. As the core link of energy system, power system is widely used, with strong regulation ability and complex control, especially with the increasing proportion of new energy and various forms of consumption. Now the power system presents the characteristics of complex nonlinearity, strong uncertainty and strong coupling. There are many limitations in traditional modeling, optimization and control technology, and artificial intelligence technology will be an effective measure to solve the control and decision-making problems of complex system. Firstly, this paper analyzes the application prospect of artificial intelligence technology in power system and the application of artificial intelligence technology in power grid operation. It establishes the prediction model of power grid operation mode, the model can help operators of power system quickly adjust the power flow to convergence state, and then greatly improve the calculation efficiency of power grid operation mode.
\end{abstract}

\section{Introduction}

The purpose of smart energy is to integrate advanced information and communication technology, intelligent control and optimization technology with modern energy production, energy consumption and user transactions, and to establish and improve energy technology and energy system meeting the requirements of ecological civilization and sustainable development, so as to form a new form of energy, which has the characteristics of automation, interaction and intelligence self-discipline control, etc. through the technological innovation in the development, utilization, mutual transformation of various types of energy, as well as the coordination, coordination, optimization and complementarity among energy networks, it can finally meeting the requirements of system, safety, cleanness and economy.The State advocates vigorously developing energy science and technology, accelerating technological innovation, making full use of renewable energy, especially breakthroughs in new energy grid connection, energy storage, micro grid and other technologies, improving the regulatory capacity of the power system, enhancing the capacity of new energy consumption, developing advanced and efficient energy-saving technologies, and realizing optimal allocation of resources, finally, the energy production and consumption will be highly automated and intelligent. As the key link of the energy system terminal consumption, the degree of intelligence of the power system will greatly affect the smooth implementation of the above technological innovation and progress. At the same time, the key link of the power system will promote the energy system to take the power system as the core. Based on the new energy related technology, the whole energy system is connected and integrated. Therefore, it is very important to develop the intelligent technology in the power system.

This paper first analyzes the application prospect of artificial intelligence technology in power system, and the artificial intelligence technology in power grid operation, establishes the model of power grid operation mode, through continuous improvement and iteration of the model in practice, it uses the model intelligence to make auxiliary decision on power grid operation mode, and helps the operation mode personnel quickly adjust the power flow to convergence state, so the calculation efficiency of power grid operation mode is greatly improved.

\section{The framework of artificial intelligence technology in smart energy}

With the change of energy structure, the new energy industry has developed rapidly. The high proportion of distributed energy penetration and large-scale access of electric vehicles make the power grid structure more complex and flexible, with the characteristics of large uncertainty, strong nonlinearity and complex coupling relationship. The power grid presents the trend of intelligent development, Its requirements for power

*Corresponding author's e-mail: 1920911787@qq.com 
system application technology tend to be efficient, simple and reliable. However, traditional technology has problems such as low reliability, lack of long-term verification and unclear mechanism. Therefore, artificial intelligence technology has become a powerful measure to solve complex power system problems by virtue of its advantages and characteristics, It is an effective tool to improve the security, reliability and economy of the new generation power system. In the research process, artificial intelligence technology is first widely used in power system scheduling, planning, trading and other fields, and its application technology analysis is shown in the figure1:

\begin{tabular}{|c|c|c|c|}
\hline \multicolumn{4}{|c|}{ The application technology in power system } \\
\hline $\begin{array}{l}\text { 1: Continuous } \\
\text { access to new } \\
\text { energy } \\
\text { 2: Different types } \\
\text { of energy } \\
\text { coupling } \\
\text { 3:Spatiotemporal } \\
\text { unsynchronism } \\
\text { of multiple } \\
\text { energy sources }\end{array}$ & $\begin{array}{l}\text { 1: Complex data } \\
\text { and high } \\
\text { dimension } \\
2 \text { :Randomness } \\
\text { 3: structural model } \\
\text { uncertainty } \\
\text { 4: strong coupling }\end{array}$ & $\begin{array}{l}\text { 1: Unable to build } \\
\text { accurate model } \\
\text { 2: Difficult to } \\
\text { optimize } \\
\text { mutivariable system } \\
\text { quantitatively } \\
\text { 3: Difficult to meet } \\
\text { requirements of high } \\
\text { intelligence }\end{array}$ & $\begin{array}{l}\text { 1: Advantages of } \\
\text { big data feature } \\
\text { analysis } \\
\text { 2: Human and } \\
\text { machine assisted } \\
\text { decision making } \\
\text { 3:Control of } \\
\text { modeless system } \\
\text { 4: Realize real-time } \\
\text { automatic control }\end{array}$ \\
\hline ᄀr & तr & 7 & 4 \\
\hline $\begin{array}{l}\text { Current situation } \\
\text { of power system }\end{array}$ & Problems & $\begin{array}{c}\text { Limitation of } \\
\text { traditional methods }\end{array}$ & $\begin{array}{l}\text { Artificial intelligence } \\
\text { technology }\end{array}$ \\
\hline
\end{tabular}

Figure 1. Application analysis of modern power system

As an important carrier of the new generation of energy reform, smart grid and energy Internet occupy the core and leading position in the development of smart energy. The main functions of energy Internet will be supported by smart grid, artificial intelligence technology, cloud computing, Internet of things, big data and mobile Internet, based on the deep integration structure of information, physics and society, The framework of energy optimization decision-making and wide area coordination is shown in Figure 2. Therefore, the integration of artificial intelligence technology and energy Internet technology will give the traditional energy network intelligent function.

In recent years, research on in-depth learning based intelligent power grid transmission line fault early warning and multi-agent based distribution network selfhealing control has been gradually launched. In the future, in the field of energy Internet, Meanwhile, artificial intelligence technology will focus on how to interact with the dynamic environment, Considering the requirement of timely, stable and safe decision-making of power system, we can design the artificial intelligence system which can learn and adapt constantly. For the research of black start power supply selection and scheme making of micro grid, how to design the personalized decision-making system with artificial intelligence technology to ensure the safety and reliability of power consumption in obstacle will also be the research focus. In addition, with the development of new generation power system, It is also necessary to explore how to comprehensively use the artificial intelligence system supporting multi-organizations to realize the energy and information integration of horizontal multi-energy complementation and vertical source network load storage coordination.

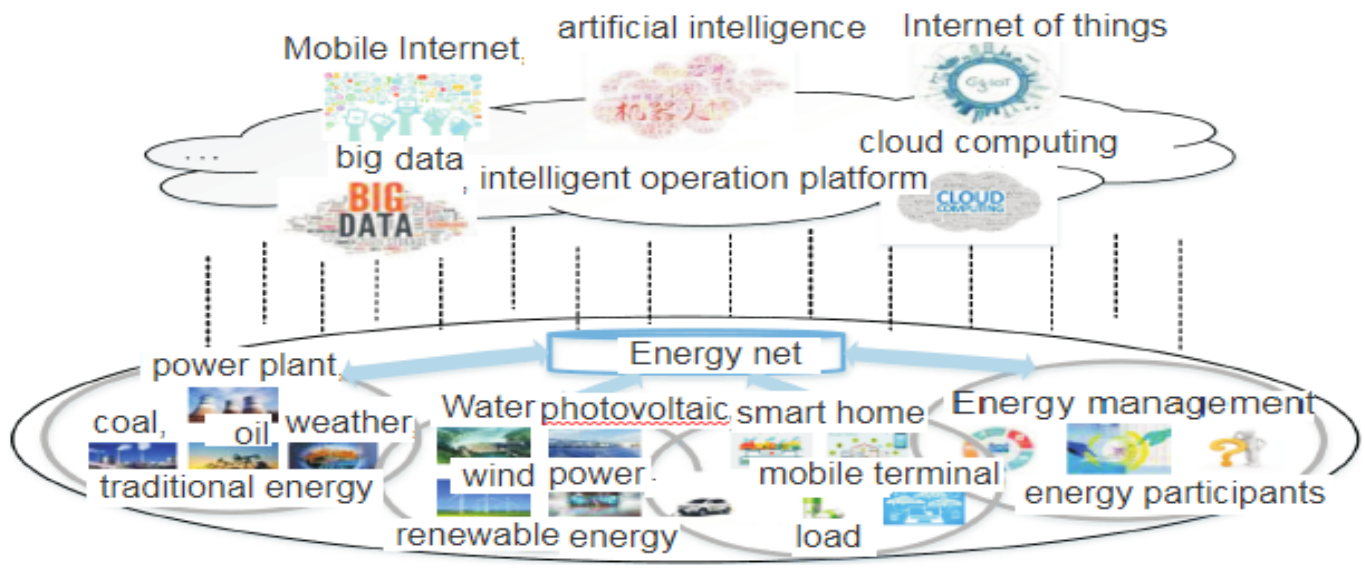

Figure 2. Application of artificial intelligence technology in smart energy 


\section{The application of artificial intelligence technology in power system}

\subsection{Current situation of power system}

The user adjusted power flow calculation mainly adjusts the active and the reactive power of the generator, the load is broken and so on. The adjusted device elements have a certain timing. It is great the significance for the user to analysis the equipment relationship by using the mass users data, and to predict the next adjustment of the equipment when the user is adjusting the flow. In recent years, with the development of neural networks, deep learning algorithms have been improved. Among them, recurrent neural network ( $\mathrm{RNN}$ ) has been applied to the modeling and prediction of sequence information because of its ability of "memory", and remarkable achievements have been achieved. But the traditional RNN has gradient dissipation problem in the process of information feedback. In order to solve the problem of long time span sequence modeling, the strategy of long and short time memory (LSTM) network is proposed by Hochreiter and Schmidhuber. the LSTM combines the historical state, current memory and current input into the gate control unit to deal with the long sequence dependence problem.

Based on the above analysis, this paper sets up the prediction model of different scale based on the LSTM network by mass adjustment of the power flow behavior data. There is a high reference value.

\subsection{The Modeling method in power system}

Since the action of adjustment power flow is similar to the timing problem, for example, when the user adjusts the active or reactive power of the generator, the equipment that should be adjusted has a certain regularity, and the LSTM model can learn the dynamic characteristics on the time series with the working principles of the forget gate, the input gate and the output gate. The different individuals often reflect different characteristics at continuous time points. For example, when the user adjusts the power flow, the generator active or reactive power or load is adjusted, the adjustment is often random. Using LSTM can effectively capturet he power system adjustment change. This paper takes every action data in the massive user adjustment data behavior as input, and adjusts the range of equipment to be adjusted at the next moment as output. Each attribute value is combined with LSTM time series connection by nonlinear transformation and predicts the probability of different states at the next time by Softmax, and determines the state of the device according to the maximum probability. The specific steps are as follows:

(1) at first, Read the device name in the user adjustment behavior data.
(2) second, See all the device names of users adjustment behavior data into a data with time series as input to LSTM.

(3) Learning and training data through LSTM model.

(4) At last, LSTM gets regular adjustment order, and when we adjust the power flow, input the current device name, the model can give the next adjustment devices. the architecture of user adjustment power flow prediction based on LSTM is shown in Figure3.

(5) the model can help the generate adjustment strategy, so it can greatly improve the calculation efficiency of power grid operation mode, the flow is shown in Figure4.

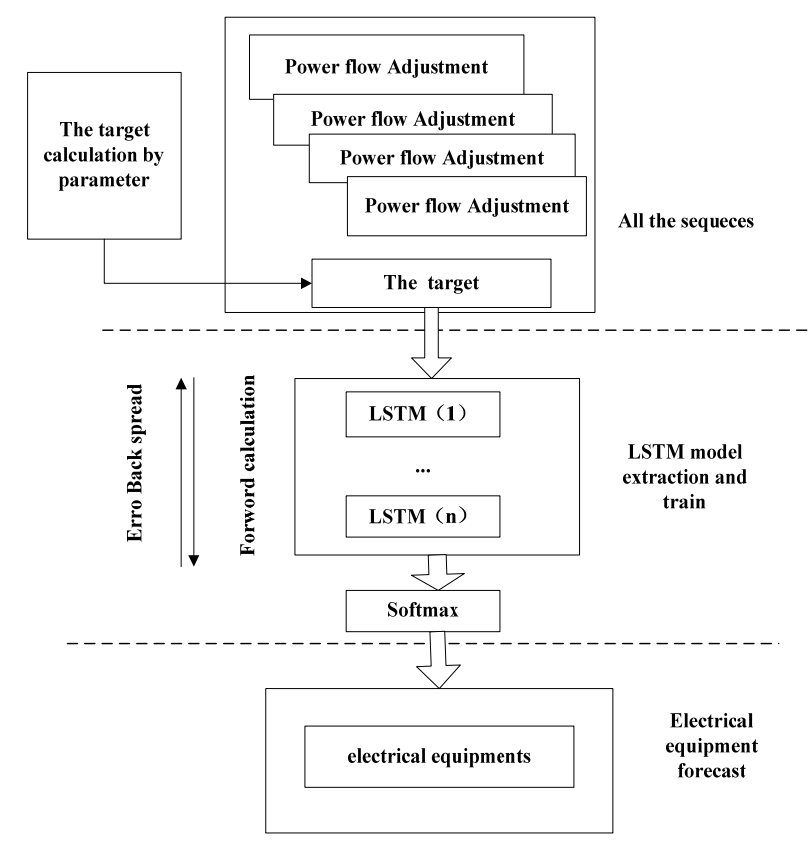

Figure 3. The LSTM Model in power flow

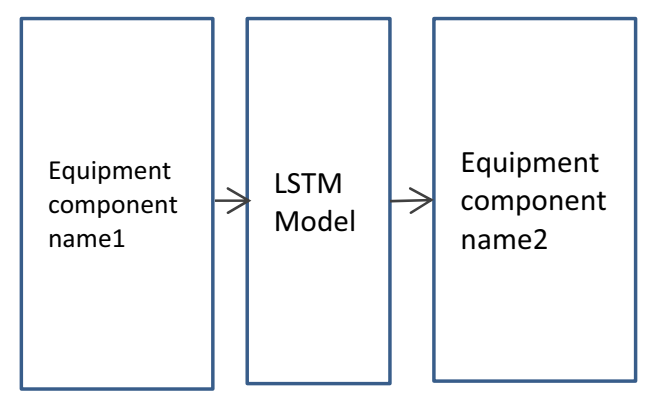

Figure 4. the flow chart of using model

\section{Conclusion}

This paper summarizes the typical application of artificial intelligence technology in the start energy, and takes the power system as an example, establishes the prediction model of power grid operation mode, uses the model intelligence to make auxiliary decision of power grid operation mode, and realizes the intelligence of power grid control. In the future, the role of artificial intelligence technology in intelligent energy will be gradually significant. 


\section{Acknowledgments}

Authors thanks to the support of Science and Technology Project of State Grid (Key Science and Technology Project of State Grid Jiangsu Electric Power Co., Ltd. J2019001).

\section{References}

1. Cai Z X. Artificial Intelligence: Principles and Applications[M]. Beijing: Tsinghua University Press, 2004: 8-20.

2. Zhou $Z$ H. Machine learning[M]. Beijing: Tsinghua University Press, 2016: 12-35.

3. Shao C, Shahidehpour M, Wang X, et al. Power system economic dispatch considering steadystate secure region for wind power[J]. IEEE Trans on Sustainable Energy,2017, 8(1): 268-278.

4. Nilsson $\mathrm{N}$ J. Artificial intelligence: A new synthesis[M]. San Fran Cisco: Morgan Kaufmann Publishers Inc, 1998:37-48.

5. Steinke G. Design aspects of access control in a knowledge base system[J]. Computers and Security, 1991, 10(7): 612-625.

6. Dang D Y. Application of artificial neural network in power system[J]. Northeastern Electric Power Technology, 1996, 1(3): 58-62

7. Sun Y, Yao P Y, et al. Cooperative task scheduling method for agent group using robust multiobjective optimization approach[J]. Control and Decision, 2016,31(11): 2045-2052.

8. Chi A, Lundén J, Koivunen V. Reinforcement learning-based plug-in electric vehicle charging with forecasted price[J]. IEEE Trans on Vehicular Technology,2017, 66(5): 3674-3684.

9. HUANG P, HIM M, HASEGAWA-JOHNSON M, et al. Joint optimization of masks and deep recurrent neural networks for monaural source separation[J]. IEEE/ACM Transactions on Audio, Speech and Language Processing, 2015, 23(12): 2136-2147.

10. Zhenlong Yuan, Yongqiang Lu. et al. Droid-Sec: deep learning in android malware detection[J], Computer Communication Review, 2014, 44(4): 371-372. 